Volume 1

Issue 1 -- Melanoma Research

Article 8

$2-28-2014$

\title{
Recurrent Acute Myocardial Infarction in Patients with Immune Thrombocytopenic Purpura
}

Fengyi Shen

Tonga Nfor

Tanvir Bajwa

Follow this and additional works at: https://aurora.org/jpcrr

Part of the Cardiology Commons

\section{Recommended Citation}

Shen F, Nfor T, Bajwa T. Recurrent acute myocardial infarction in patients with immune thrombocytopenic purpura. J Patient Cent Res Rev. 2014;1:41-45. doi: 10.17294/2330-0698.1010

Journal of Patient-Centered Research and Reviews (JPCRR) is a peerreviewed scientific journal whose mission is to communicate clinical and bench research findings, with the goal of improving the quality of human health, the care of the individual patient, and the care of populations.

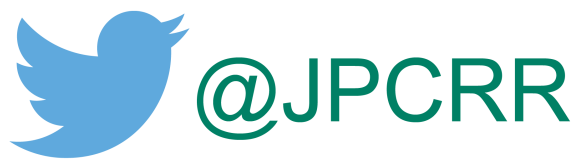




\title{
Recurrent Acute Myocardial Infarction in Patients with Immune Thrombocytopenic Purpura
}

\author{
Fengyi Shen, MD, ${ }^{1}$ Tonga Nfor, MD, ${ }^{2}$ Tanvir Bajwa, $\mathrm{MD}^{2}$ \\ ${ }^{1}$ Department of Internal Medicine and ${ }^{2}$ Department of Cardiology, Aurora Health Care, Milwaukee, WI
}

\begin{abstract}
Immune thrombocytopenic purpura (ITP), also known as idiopathic thrombocytopenic purpura, is an acquired immune-mediated disease of adults and children characterized by a transient or persistent decrease of platelets and, depending upon the degree of thrombocytopenia, an increased risk of bleeding. The use of standard treatments for acute myocardial infarction (AMI), such as antiplatelet agents and anticoagulants, pose serious problems in patients with ITP due to the potential higher risk of bleeding complications. There are no current guidelines available for management of ITP patients with AMI. In this brief review of the limited available literature, we discuss the proposed pathophysiological link between ITP and arterial thrombosis and the challenging medical and interventional treatment of these patients. (J Patient-Centered Res Rev. 2014;1(1):41-45.)
\end{abstract}

\section{Keywords}

myocardial infarction, purpura, thrombocytopenia, percutaneous coronary intervention, anticoagulation

\section{Introduction}

Immune (idiopathic) thrombocytopenic purpura (ITP), an acquired immune-mediated disease of adults and children characterized by a transient or persistent decrease of platelets, poses an increased risk of bleeding among affected patients. ${ }^{1,2}$ The additional risk varies according to the degree of thrombocytopenia. ITP patients who also endure acute myocardial infarction (AMI), are rarely encountered. The standard treatments for AMI, including antiplatelet agents and the necessity of the administration of anticoagulants and antiplatelet agents during and following a percutaneous coronary intervention (PCI) procedure, is the major concern for this group of patients. Thrombolytic therapy is contraindicated in ITP because of the high risk of bleeding. There are no current guidelines for the management of AMI

Correspondence: Fengyi Shen, MD

c/o 1020 N. 12th Street, Suite 4180, Milwaukee, WI 53233

Email: fyshen99@yahoo.com in thrombocytopenic patients. In this report, several possible etiologies for thrombosis in ITP are discussed in light of a 58-year-old female with chronic ITP who developed recurrent AMI secondary to thrombosis. This case, along with the limited available literature, suggests that a modified treatment strategy, carefully combining PCI with antiplatelet and anticoagulant therapy, can be administered successfully for AMI in ITP patients.

\section{Illustrative Case}

A 58-year-old female with a history of chronic ITP with multiple exacerbations, complicated by diabetes mellitus and hypertension secondary to long-term steroid use, presented with shortness of breath and chest pain over 24 hours. The patient was initially diagnosed with ITP in 1988 and had been taking prednisone 5-10 mg daily nearly continuously. During that time, she had experienced several ITP exacerbations which were typified by gingivorrhagia and petechiae on her legs (with extremely low platelet counts, once reaching a nadir of $3 \times 10^{9} / \mathrm{L}$ ). The flares were treated with rituximab, IV immunoglobin, and/or high doses of prednisone. A splenectomy had also been performed secondary to repetitive episodes of thrombocytopenia. One week prior to this hospitalization, the patient had a severe acute ITP exacerbation, with a platelet count minimum of $9 \times 10^{9} / \mathrm{L}$ and was treated with a high dose of prednisone, plus rituximab, for three days before presentation. Laboratory data obtained on admission included a platelet count of $100 \times 10^{9} / \mathrm{L}$, an elevated troponin I of $6.6 \mu \mathrm{g} / \mathrm{L}$ and a CK-MB of 63. Admission vital signs were stable. An EKG revealed ST segment elevations and small Q-waves in leads I and aVL, associated with ST depressions in the inferior leads (Fig. 1a). ST-elevation myocardial infarction (STEMI) was diagnosed and the patient was immediately referred to the cardiac catheterization laboratory. Subsequent coronary angiography revealed a clot in the midleft anterior descending artery (LAD) which was deemed the likely culprit lesion for her myocardial infarction (Fig. 2a). Aspiration thrombectomy was performed with successful restoration of brisk flow throughout the entire LAD. Chest pain and ST-segment changes subsequently improved. There was no lesion that required stenting, and all coronary arteries were free of underlying atherosclerotic disease; intravenous 

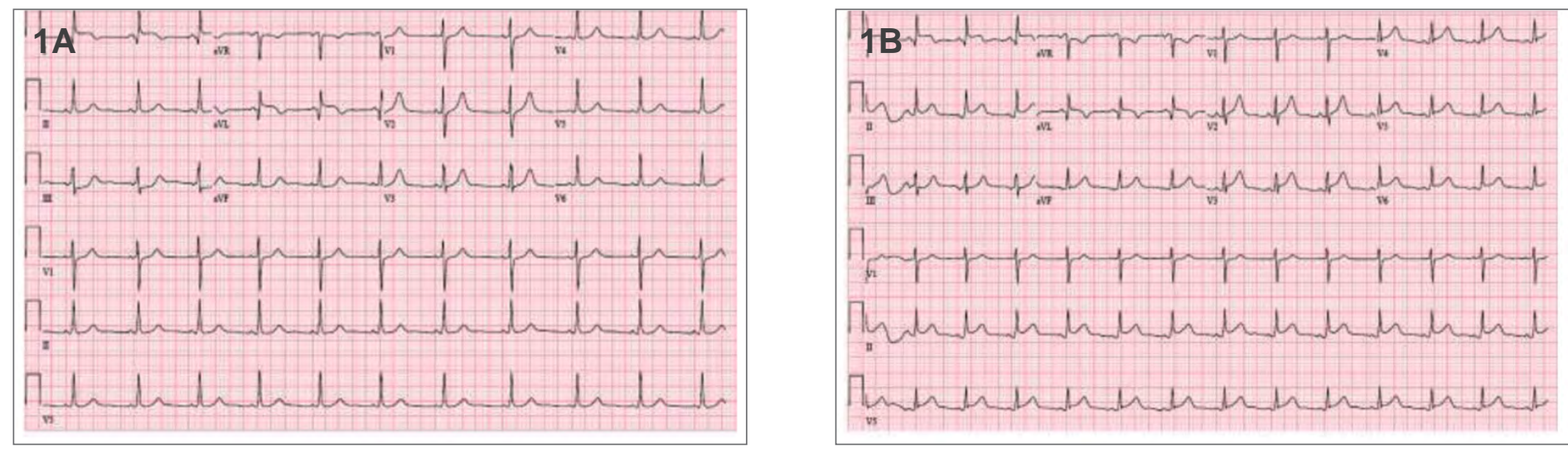

Figure $1 \mathbf{a}$ and $\mathbf{1 b}$. Electrocardiogram tracings recorded on the day of admission (a) and on the day of recurrent MI (b).

(IV) heparin bolus (5,000 units), without further infusion, was given during the procedure and eptifibatide was bolused at $180 \mathrm{mcg} / \mathrm{kg}$ followed by continuous infusion at $2 \mathrm{mcg} / \mathrm{kg} / \mathrm{min}$ for 12 hours. Aspirin $325 \mathrm{mg}$ and clopidogrel $75 \mathrm{mg}$ were administered orally before and daily after the procedure.

Within 16 hours, the patient developed recurrent severe chest pain associated with new ST-segment elevations in the lateral and anterior leads (Fig. 1b), as well as progressive cardiogenic shock. Repeat angiogram was pursued and demonstrated a diffusely thrombosed LAD with heavy clot burden and minimal flow distally (Fig. 3a). Extensive aspiration thrombectomy was again attempted. Flow improved in the proximal LAD, but there was only mild improvement distally with some residual embolization (Fig. 3b). Intracoronary adenosine was then given without improvement in blood flow. During the PCI, eptifibatide and heparin were reinitiated. This time the heparin infusion was continued for 16 hours post procedure. After completion of eptifibatide, the patient received a bivalirudin drip for three days following the catherization. The patient was discharged home in stable condition after 11 days of hospitalization without bleeding or thrombocytopenic complications. Her platelet count at discharge was $475 \times 10^{9} / \mathrm{L}$. One month later, she was readmitted for unstable angina without recurrent coronary artery thrombosis detected on angiography. She subsequently received an implantable cardioverter defibrillator because of a persistent $25 \%$ cardiac ejection fraction by echocardiography.

Considering her recurrent arterial thrombosis without predisposing anatomical lesions, extensive coagulation tests were performed including lupus anticoagulant, anticardiolipin antibody, beta-2 glycoprotein antibodies, protein C, protein S, homocysteine and factor VII. All tests were negative.

\section{Review of Acute Myocardial Infarction in Patients} with Immune Thrombocytopenic Purpura

Although ITP usually causes bleeding and is not considered a prothrombotic state, it may be associated with increased risk of thrombotic events including myocardial infarction, ischemic stroke, ${ }^{3}$ and venous thrombosis. Thrombotic complications have been reported to occur during or after treatment when platelet counts begin to rise ${ }^{4,5}$ and even during the severe to moderate thrombocytopenia phase. ${ }^{6}$ However, the reported incidence of myocardial infarction in patients with chronic thrombocytopenia is low. ${ }^{7}$

\section{Possible Etiology of Thrombosis in ITP}

The mechanism of arterial thrombosis in ITP patients is still unclear, but several hypotheses exist:

- One theory is that an antibody reaction shatters the platelets and releases platelet microparticles (PMPs), which are highly thrombogenic. PMPs may protect against bleeding in thrombocytopenic states, but they may also precipitate thrombosis. In this case, the synergistic action of prothrombotic factors in ITP may override the protective effect of thrombocytopenia.

- In ITP patients, platelets are larger, younger, and thus more active. The treatment in ITP may cause the platelet count to rise very rapidly.

- Some therapies can cause thrombotic complications, including splenectomy. Splenectomy, in turn, may cause portal vein thrombosis, pulmonary embolism and deep vein thrombosis. ${ }^{8}$

- Steroid therapy is known to induce metabolic changes, as well as a hypercoagulable state which may contribute to the clinical scenario of thrombogenesis intravenous immunoglobulin (IVIG). This can increase blood viscosity, activating platelets or causing vasospasm. ${ }^{9}$

- Danazol is sometimes used in treating ITP patients and has been noted to be associated with arterial thrombosis. ${ }^{10}$ 

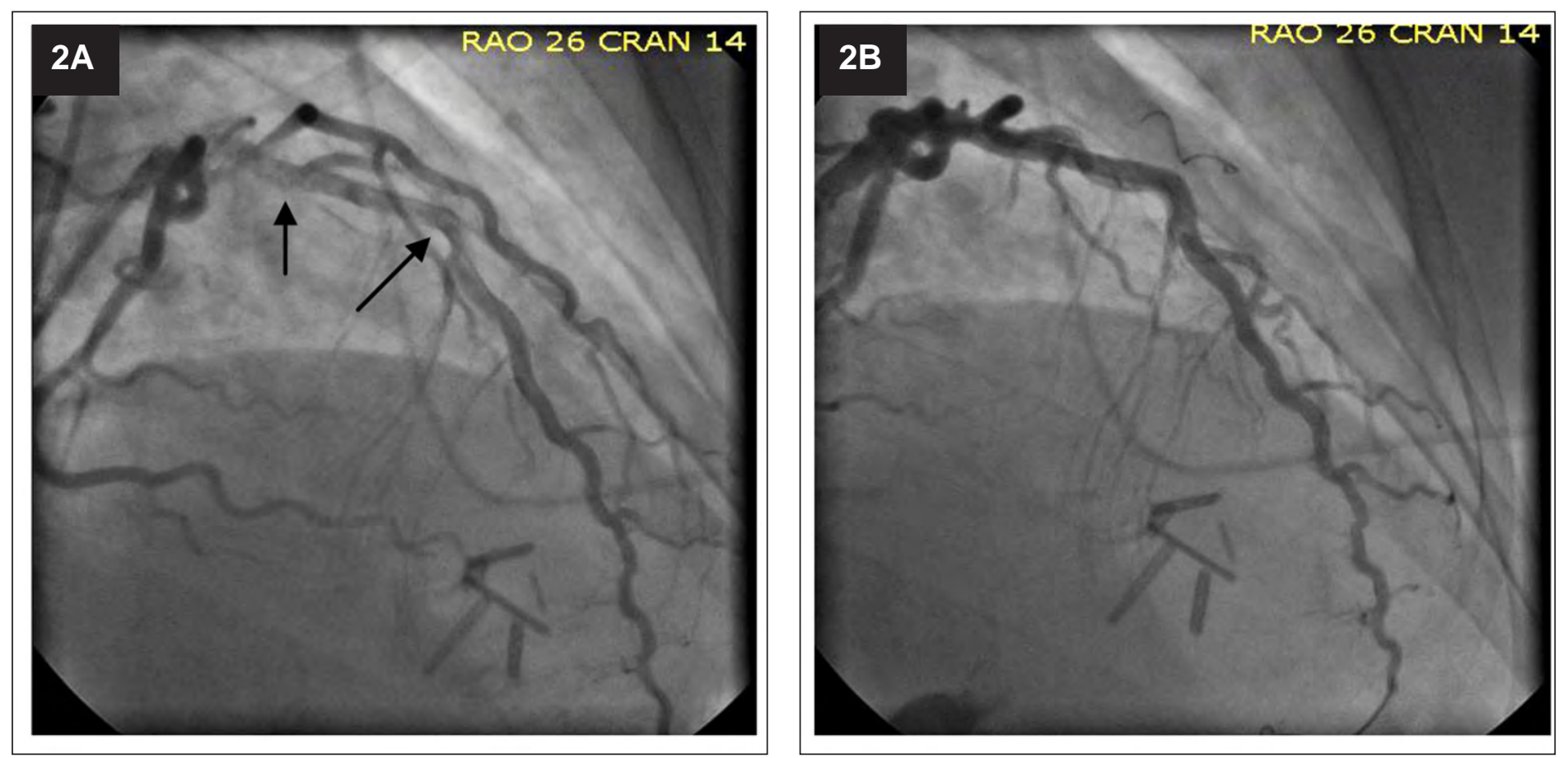

Figure $\mathbf{2 a}$ and $\mathbf{2 b}$. First selective left coronary angiogram showing thrombotic lesions in the LAD (a) and angiographic result after thrombectomy with restored blood flow (b).
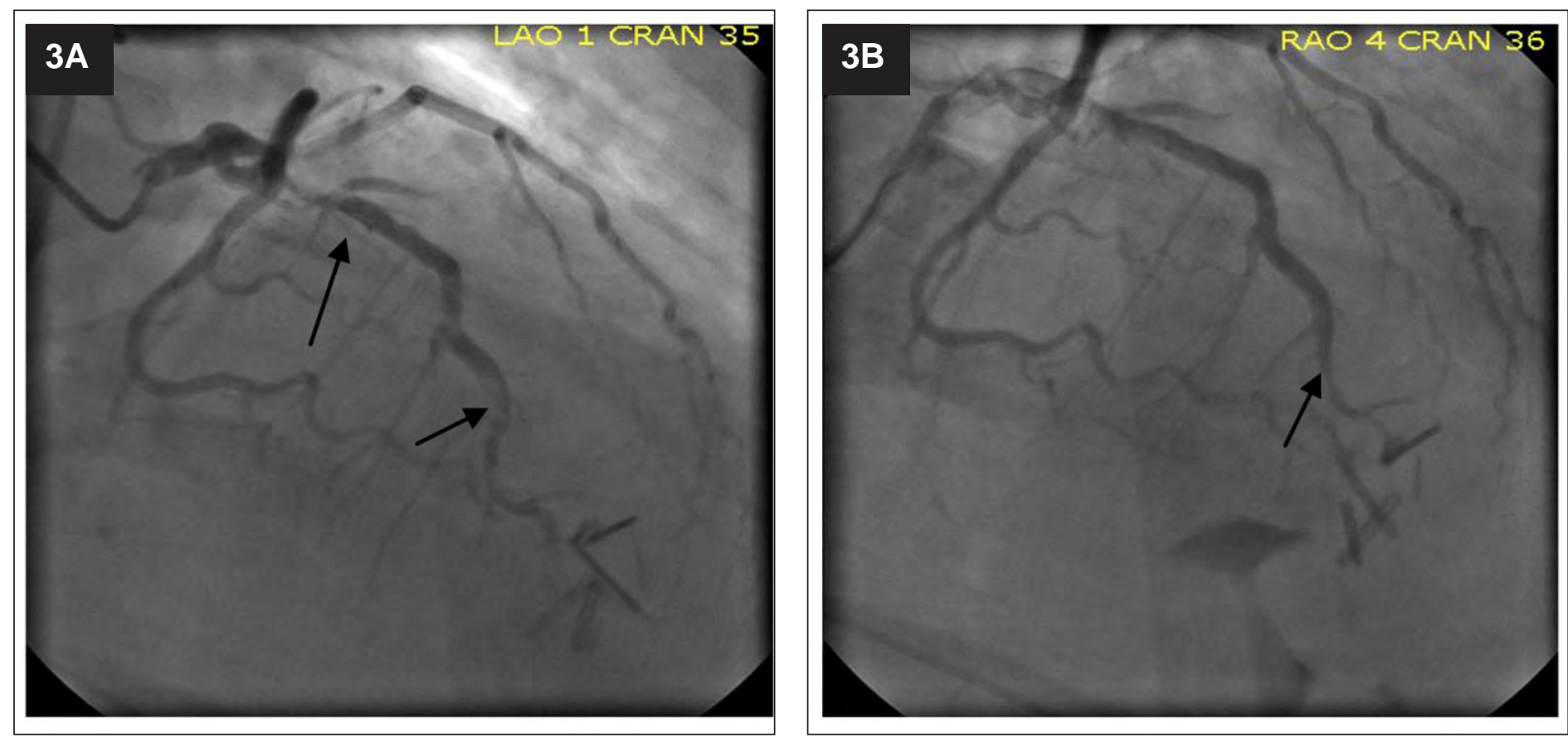

Figure $\mathbf{3 a}$ and $\mathbf{3 b}$. Second selective second left coronary angiogram showing diffusely thrombosed LAD with heavy clot burden (a) and angiographic result after re-thrombectomy with mild improvement of the blood flow (b).

- Another possible etiology of the thrombotic events in ITP patients is that the autoantibodies in ITP directed against antigens present on both platelets and endothelial cells might induce endothelial damage, thereby causing thrombus formation and embolic occlusion of the artery. ${ }^{6}$

- A considerable percentage of patients with ITP also have antiphospholipid antibodies (aPL), which have been associated with thrombotic events in other patients. The exact frequency and clinical significance of aPL in ITP patients, however, is still controversial. In one study of patients with newly diagnosed ITP and platelet counts $<50 \times 10 \%$, aPL were found in $26 \%$, but their presence was not associated with clinical manifestations of ITP. ${ }^{11}$ As noted, the patient in our illustrative case was negative for aPL. 


\section{Challenging Treatment of AMI in ITP Patients}

Management of AMI in ITP patients is still controversial. Thrombolytic therapy for the treatment of AMI is contraindicated in ITP patients due to the high risk of bleeding diathesis. ${ }^{12} \mathrm{PCI}$ is challenging, but it is considered the most effective approach to treat these patients. The necessity of stent implantation, as well as the administration of anticoagulants and antiplatelets during and following the procedure, is the major concern related to the PCI. Performance of PCI in a patient with ITP presents a unique situation in which platelet function needs to be inhibited sufficiently to perform PCI safely, but not to the extent that bleeding complications occur. ${ }^{13}$

We were able to find six cases reported in the literature of primary PCI for AMI patients with ITP. Fuchi et al. ${ }^{14}$ reported a 71-year-old ITP patient with AMI who underwent balloon angioplasty. The patient developed LAD reinfarction and a second primary PCI was performed, later complicated by a large hematoma at the femoral arterial access site. The patient received IVIG and methylprednisolone, which resulted in bleeding cessation. Gracia et al. ${ }^{15}$ reported an ITP patient who underwent PCI for total LAD occlusion. He received heparin prophylaxis and combined antiplatelet therapy. Fong et al. ${ }^{16}$ reported a case of acute coronary syndrome in a 71-year-old female with ITP. The patient received IVIG premedication and underwent PCI through the radial artery. The patient tolerated seven weeks of clopidogrel treatment without complications. Caputo et al. ${ }^{17}$ reported a 62-year-old refractory ITP patient with a platelet count of $3 \times 10^{9} / \mathrm{L}$ who received stent placement in the LAD. This was followed by four weeks of aspirin and two weeks of clopidogrel without bleeding complications. Stouffer et al. ${ }^{13}$ reported a 77-year-old man with ITP and platelet count of $70 \times 10^{9} / \mathrm{L}$ who underwent two separate cardiac catherization procedures due to obstructive disease of the left circumflex and restenosis at the site of the previous lesion. Eptifibatide, clopidogrel and an intracoronary stent were administered without complications. Another case was reported by Kim et al. ${ }^{18}$ regarding a 47-year-old woman with chronic ITP who was diagnosed with inferior AMI and treated successfully by primary PCI. Heparin was given during the PCI and aspirin and clopidogrel were instituted after the procedure.

In our illustrative case, the patient had no atheromatous process detected in her coronary arteries, therefore, stent placement was not warranted. For ITP patients with underlying coronary artery disease, available data suggests that baremetal stents may be preferrable to drug-eluting stents, because of the shorter duration of dual antiplatelet therapy (aspirin plus clopidogrel) required. However, there is no direct data comparing the safety of the two stent types in ITP. ${ }^{19}$

Heparinoids, including unfractionated heparin (UFH) and fondaparinux, have been used successfully during PCI in patients with ITP. There is no optimal dose of heparin suggested in such patients. However, the standard weightadjusted dose, or smaller dose of heparin plus IVIG therapy during the thrombocytopenic phase, ${ }^{18}$ has been recommended. Our patient was given heparin during the first PCI, but the infusion was not continued after the first procedure secondary to bleeding concerns. She experienced reinfarction within 24 hours, which raises the question of whether a continuous infusion of heparin might have been able to prevent this. Neskovic et al. suggested fondaparinux can be used with a half dose of UFH, so as to minimize the risk of heparin-induced thrombocytopenia (HIT). In addition, fondaparinux, unlike UFH and low molecular weight heparin, has no effect on platelet function and may thus decrease the risk of serious bleeding in thrombocytopenic patients. ${ }^{20}$ The addition of UFH to fondaparinux is recommended due to an increased risk of catheter thrombosis that has been reported when using fondaparinux alone during PCI.

The direct thrombin inhibitors are considered to be a valuable option for patients with ITP undergoing PCI. Bivalirudin is the only agent approved to use for anticoagulation during PCI in patients with HIT, although other direct thrombin inhibitors (lepirudin and argatroban) have also been used. ${ }^{21}$ The administration of glycoprotein IIb/IIIa inhibitors, such as eptifibatide, are indicated during and following PCI, but they have been reported to produce severe thrombocytopenia in some ITP patients while they were well-tolerated in other reports. ${ }^{14,22}$ Based on the above observations, we switched from eptifibatide to bivalirudin for our patient. Neither eptifibatide nor bivalirudin led to any bleeding or thrombocytopenic complications in our case.

Moreover, it is recommended that dual antiplatelet therapy with aspirin and clopidogrel should be prescribed for a shorter period of time to allow stent endothelialization. Our patient was given aspirin and clopidogrel before and after the procedure even without stent implantation.

\section{Conclusion}

The mechanism of thromboembolism in ITP patients is still unclear, but several hypotheses exist. Thrombolytic therapy is contraindicated in AMI in ITP patients. Clinicians caring for patients with AMI in the face of ITP should consider that primary PCI may be an effective and safe therapeutic 
treatment. Antiplatelet and anticoagulation therapy can be used cautiously during and after PCI without bleeding or thrombocytopenic complications. Also, dual antiplatelet therapy (aspirin plus clopidogrel) can be well-tolerated in these patients if thrombocytopenia is not severe.

\section{Acknowledgments}

\section{Contributors}

Dennis J. Baumgardner, MD, for manuscript suggestions.

\section{Funders}

None.

\section{Prior presentation of data}

Fengyi Shen: Recurrent Acute Myocardial Infarction in a Patient with Immune Thrombocytopenic Purpura. American College of Physicians Wisconsin Chapter Annual Scientific Meeting, 9/10/2011

\section{Conflicts of Interest}

Authors do not have commercial or other associations that might pose a conflict of interest (e.g. pharmaceutical stock ownership, consultancy).

\section{References}

1 Cooper N, Bussel J. The pathogenesis of immune thrombocytopaenic purpura. Br J Haematol. 2006;133:364-74.

2 Cines DB, Blanchette VS. Immune thrombocytopenic purpura. N Engl J Med. 2002;346:995-1008.

3 Rhee HY, Choi HY, Kim SB, Shin WC. Recurrent ischemic stroke in a patient with idiopathic thrombocytopenic purpura. $J$ Thromb Thrombolysis. 2010;30:229-32.

4 Paolini R, Fabris F, Cella G. Acute myocardial infarction during treatment with intravenous immunoglobulin for idiopathic thrombocytopenic purpura. Am J Hematol. 2000;65:177-8.

5 Elkayam O, Paran D, Milo R, Davidovitz Y, Almoznino-Sarafian D, Zeltser D, Yaron M, Caspi D. Acute myocardial infarction associated with high dose intravenous immunoglobulin infusion for autoimmune disorders. A study of four cases. Ann Rheum Dis. 2000;59:77-80.

6 Fruchter O, Blich M, Jacob G. Fatal acute myocardial infarction during severe thrombocytopenia in a patient with idiopathic thrombocytopenic purpura. Am J Med Sci. 2002;323:279-80.

7 Juhan-Vage I. Haemostatic parameters and vascular risk. Atherosclerosis. 1996;124 Suppl:S49-55.

8 Mohren M, Markmann I, Dworschak U, Franke A, Maas C, Mewes S, Weiss G, Jentsch-Ullrich K. Thromboembolic complications after splenectomy for hematologic diseases. Am J Hematol. 2004;76:1437.

9 Emerson GG, Herndon CN, Sreih AG. Thrombotic complications after intravenous immunoglobulin therapy in two patients. Pharmacotherapy. 2002;22:1638-41.

10 Alvarado RG, Liu JY, Zwolak RM. Danazol and limb-threatening arterial thrombosis: two case reports. J Vasc Surg. 2001;34:1123-6.

11 Pierrot-Deseilligny, Despujol C, Michel M, Khellaf M, et al. Antiphospholipid antibodies in adults with immune thrombocytopenic purpura. Br J Haematol. 2008;142(4):638-43.
12 Antman EM. ST-elevation myocardial infarction: management. In: Zipes DP, Libby P, Bonow RO, Braunwald E (eds.), Heart Disease: Textbook of Cardiovascular Medicine, 7th ed. vol. 2, Philadelphia: Saunders;2005:1167-1226.

13 Stouffer GA, Hirmaerova J, Moll S, Rubery B, Napoli M, Ohman M, Simpson R. Percutaneous coronary intervention in a patient with immune thrombocytopenia purpura. Catheter Cardiovasc Interv. 2004;61:364-7.

14 Fuchi T, Kondo T, Sase K, Takahashi M. Primary percutaneous transluminal coronary angioplasty performed for acute myocardial infarction in a patient with idiopathic thrombocytopenic purpura. Jpn Circ J. 1999;63:133-6.

15 Gracia MC, Cebollero IC, Lezcano JS, Osuna GG, Miguel JA, Peralta LP. Invasive treatment performed for acute myocardial infarction in a patient with immune thrombocytopenic purpura. Int J Cardiol. 2008;127:e183-5.

16 Fong MC, Chen KC, Leu HB, Chen LC. Coronary revascularization in a patient with immune thrombocytopenic purpura. J Chin Med Assoc. 2006;69;436-8.

17 Caputo RP, Abraham S, Churchill D. Transradial coronary stent placement in a patient with severe idiopathic autoimmune thrombocytopenic purpura. J Invasive Cardiol. 2000;12:365-8.

18 Kim JH, Park KU, Chun WJ, Kim SH, Nah DY. Primary percutaneous coronary intervention for acute myocardial infarction with idiopathic thrombocytopenic purpura: a case report. J Korean Med Sci. 2006;21:355-7.

19 Moretti C, Teresa Lucciola M, Morena L, et al. Idiopathic thrombocytopenic purpura and percutaneous coronary stenting: a dangerous duo? Int J Cardiol. 2008;130(3):e96-7.

20 Neskovic AN, Stankovic I, Milicevic P, et al. Primary PCI for acute myocardial infarction in a patient with idiopathic thrombocytopenic purpura. A case report and review of the literature. Herz. 2010;35(1):43-9.

21 Weitz JI, Buller HR. Direct thrombin inhibitors in acute coronary syndromes: present and future. Circulation. 2002;105:1004-11.

22 Méndez TC, Diaz O, Enríquez L, et al. Severe thrombocytopenia refractory to platelet transfusions, secondary to abciximab readministration, in a patient previously diagnosed with idiopathic thrombocytopenic purpura. A possible etiopathogenic link. Rev Esp Cardiol. 2004;57:789-91.

(c) 2014 Aurora Health Care, Inc. 